\title{
Estimates from 11 countries show the significant impact of COVID-19 excess mortality on the incidence of family bereavement
}

Mallika Snyder I snyder@demogr.mpg.de Diego Alburez-Gutierrez I alburezgutierrez@demogr.mpg.de Iván Williams

Emilio Zagheni I office-zagheni@demogr.mpg.de 


\title{
Estimates from 11 countries show the significant impact of COVID-19 excess mortality on the incidence of family bereavement
}

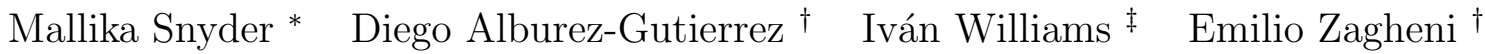

February 16, 2022

Classifications: Social Sciences (Major), Demography (Minor)

Keywords: COVID-19, excess mortality, bereavement

\begin{abstract}
Excess mortality associated with the COVID-19 pandemic has led many to experience the loss of family members, with significant negative outcomes. We quantify the extent to which these population-wide rates of kin loss represent a departure from levels expected in the absence of COVID19 excess mortality, and consider which demographic groups are most likely to be affected. Results for 11 countries indicate dramatic increases in excess kin loss associated with excess mortality, and follow a generational pattern consistent with COVID-19 mortality risk by age. During periods of high excess mortality, the number of younger individuals losing a grandparent increased by up to 851 per 100,000, or 1.43 times expected levels (for women aged 30-44 in the UK in May 2020), while the number of older individuals losing a sibling increased by up to 458 per 100,000 or 1.36 times (for women aged 65 and over in Bulgaria in December 2020). Our monthly multi-country estimates of excess kin loss complement existing point estimates of the number of individuals bereaved by COVID-19 mortality (Verdery et al., 2020; Kidman et al., 2021; Hillis et al., 2021) and highlight the role of heterogeneous excess mortality in shaping country experiences.
\end{abstract}

\section{Introduction}

As excess mortality associated with the COVID-19 pandemic is better estimated and understood 1, 2, less is known about how this is reflected in kin loss by survivors of the pandemic. The scale of pandemic-associated bereavement is significant. Each United States COVID-19 death leaves nine close kin bereaved [3], including 0.078 children aged 0 to 17 experiencing parental bereavement 4], and the pandemic has resulted in over one million new orphans under age 18 globally as of May 2021 5. This will have important population health and welfare implications given the known negative consequences of kin loss, including declines in physical and mental health and the loss of social and economic support 6. 7, 8. What remains to be better understood, however, is how these increases in population-wide bereavement shape individual-level risks of losing relatives - by how much does the incidence of kin loss increase in periods of high excess mortality, and which demographic groups are likely to be affected? Answering this question is key for better understanding the lived experience of pandemic excess mortality for those who survive it.

In this study, we complement estimates of numbers of bereaved individuals associated with COVID-19 mortality by extending a set of existing demographic microsimulations [9] to consider how pandemicassociated excess mortality affected the number of individuals experiencing losses in their close family networks, across 11 countries, each month between March 2020 and June 2021. Our estimates, which demonstrate a generational pattern of kin loss, show consistent increases across countries in the numbers of younger individuals losing a grandparent, and of older individuals losing a sibling. Moreover, our results highlight the role of heterogeneity in excess mortality and population and kinship structure in shaping levels of family bereavement.

\section{Results}

To estimate our outcome measure, termed "excess bereavement", we consider individuals that survive to at least July 2021. Some of these individuals experienced the loss of a certain type of relative between March 2020 and June 2021, but would not have in the absence of COVID-19 mortality. Our approach

\footnotetext{
*Department of Demography, University of California, Berkeley, California 94720. Email: mallikasnyder@berkeley.edu

$\dagger$ Lab of Digital and Computational Demography, Max Planck Institute for Demographic Research, Rostock, Germany 18057

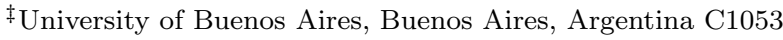


focuses on the average additional losses associated with a COVID-19 excess mortality scenario over those associated with a scenario in which only seasonally adjusted mortality in line with previous years had occurred (a counterfactual baseline scenario). We divide the number who lost kin in each month by the total population of survivors (irrespective of their kin availability) and use it to calculate the absolute difference in the number of individuals who experience loss per 100,000 in a given month and age group $\left(E B_{x, t}\right)$ across the two scenarios. This measure, which is comparable across different countries and types of kinship, is expressed in equation 1, where $N$ is the number of individuals in age group $x$ (as of February 2020) who survive to July 2021, with subscripts $b$ denoting the number who lose kin in a particular month $t$ between March 2020 and June 2021, and 0 denoting the counterfactual case:

$$
E B_{x, t}=\left(\frac{N_{b, x, t}}{N_{x}}-\frac{N_{b, x, t, 0}}{N_{x, 0}}\right) \times 100,000
$$

We plot values of $E B_{x, t}$ for a set of countries with varied mortality experiences associated with COVID-19 in Figure 1a (note the different scales between panels). As expected, the concentration of COVID-19 excess mortality among older individuals is reflected in accompanying increases in numbers of younger individuals aged 15-44 losing a grandparent, and numbers of older individuals aged 65 and above losing a sibling. Country-specific trends in COVID-19 mortality, such as mortality 'waves', are mirrored in trends in excess bereavement. Notable in Figure 1 a are short periods of negative excess bereavement following spikes in excess mortality. This suggests that these spikes may have reflected an acceleration of kin mortality, with deaths occurring sooner than otherwise expected.

Comparing Sweden, which saw high excess mortality early in the pandemic, and Norway, which saw lower mortality than might have been expected, reveals the role of excess mortality in shaping experiences of countries with otherwise very similar age-sex and kinship structures. Sweden saw significant increases in kin loss during the period, while Norway saw very low or negative changes in the number of individuals experiencing bereavement. The similarity in counterfactual rates of loss across not only Sweden and Norway, but all countries considered in Figure 1b, further indicates the importance of excess mortality even when considering countries with more varied demographic structures.

Kin loss would have been high for some groups even in the absence of COVID-19 mortality. For example, 636 (standard error (SEM) across 100 paired simulations of 21) per 100,000 UK females aged 30-44 would have experienced the loss of a grandparent in March 2020 under a baseline scenario. However, very high excess mortality associated with the pandemic resulted in kin loss more than double baseline levels; for example, by May 2020, over 851 (SEM 35) per 100,000 more individuals in this same group lost a grandparent, 1.43 times higher than the expected 703 (SEM 22) per 100,000. A comparably large increase in bereavement for older individuals was also observed, with 458 (SEM 16) per 100,000 or 1.36 times more females in Bulgaria aged 65+ losing a sibling in December 2020 than the expected 378 (SEM $9)$ per 100,000. The scale of loss associated with the pandemic is brought out even more clearly in Figure 1c. which multiplies $E B_{x, t}$ values from Figure 1a by country populations in 2020 to highlight the large total number of individuals as of July 2021 who may have lost relatives as a result of COVID-19 excess mortality.

\section{Discussion}

With estimates showing in some cases a doubling of kin loss risks over expected levels, our results help put into context the staggering toll of excess mortality and bereavement associated with COVID19 and highlight which groups are most likely to be affected. They also highlight the importance of excess mortality in shaping country experiences: the countries in our sample would have had similar population-adjusted projections of kin loss in the absence of COVID-19, but diverged considerably based on COVID-19 excess mortality.

This similarity of counterfactual kin loss reflects other similarities between countries in our sample, which are high-income countries for which excess mortality data is readily available. We might see different impacts of similar levels of excess mortality for younger populations with more varied kinship structures, such as in countries in the Global South. Further work is needed to understand how excess mortality and demographic structure shape country-level excess bereavement.

We present a lower-bound estimate, as the pandemic continues to take its toll. Furthermore, the quantities we estimate are averages at the country level: levels of excess bereavement are likely to be considerably higher in hard-hit sub-national populations, and among individuals with larger families. In spite of this, average estimates of excess bereavement remain substantially high. Future work will be needed to estimate the impact of later pandemic mortality on both the magnitude of excess bereavement and longevity of survivors themselves. 


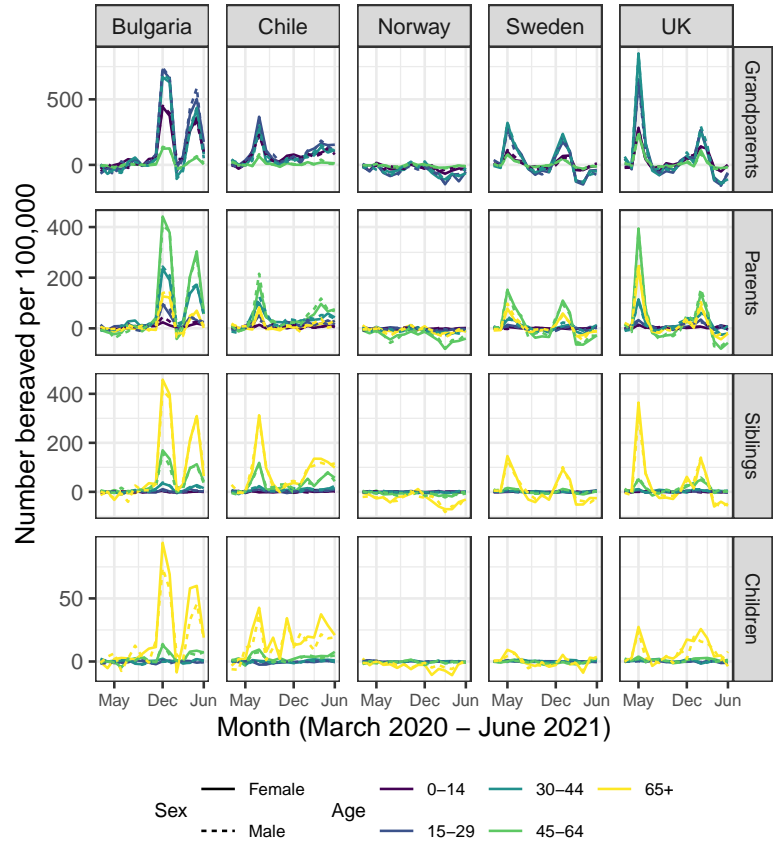

(a) Excess Bereavement

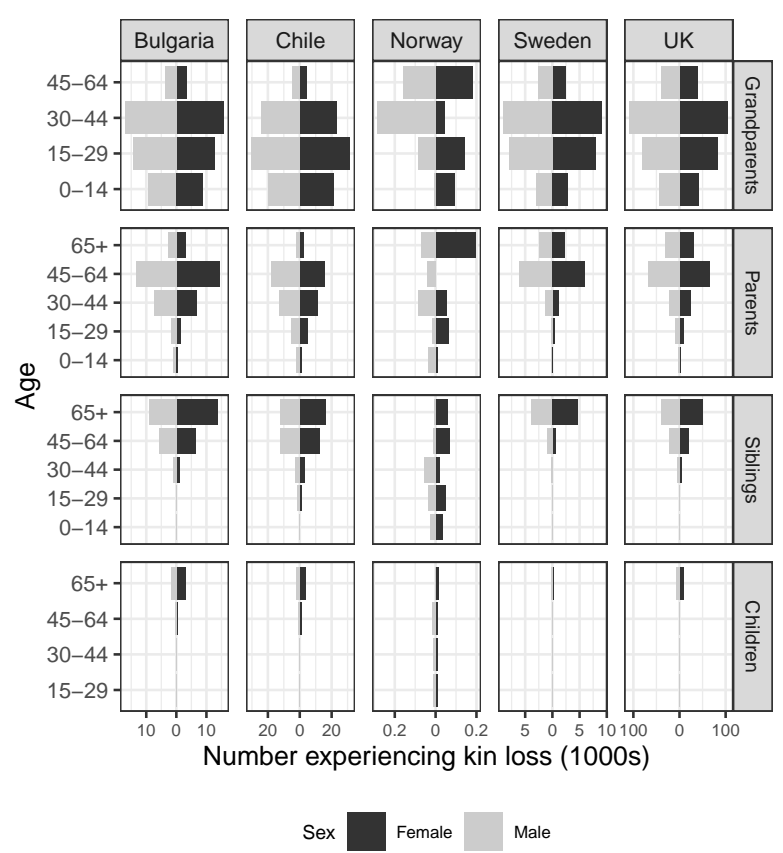

(c) Populations Experiencing Excess Bereavement

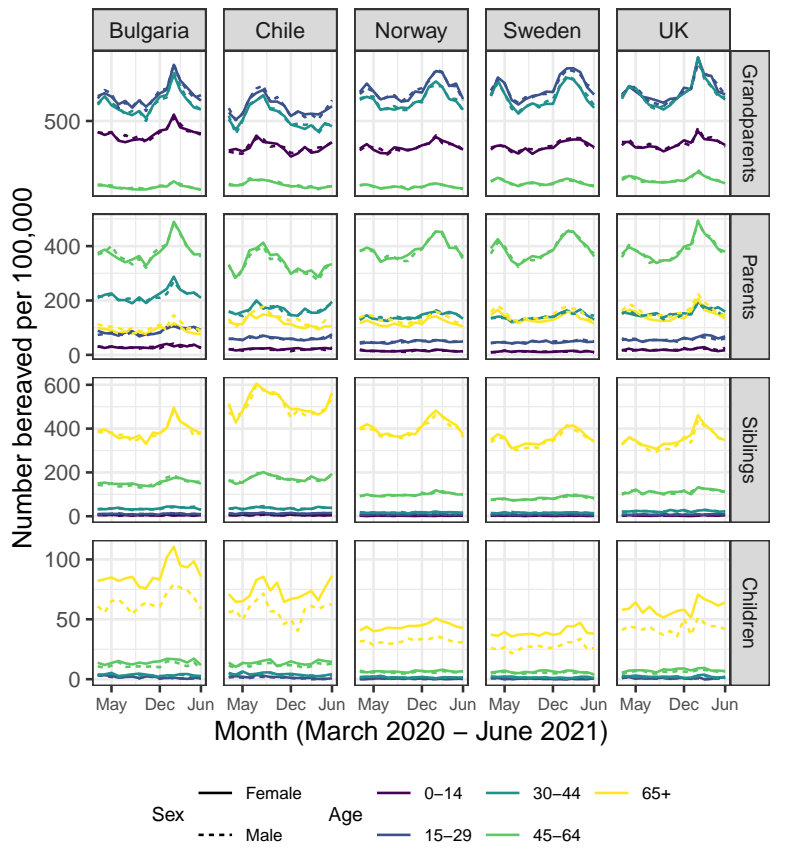

(b) Counterfactual Bereavement

Figure 1: Estimates of monthly excess bereavement $\left(E B_{x, t}\right.$, Figure 1a) and counterfactual bereavement (Figure 1b) in multiple countries by age and sex of the bereaved individual (Ego), as well as kin relation. Figure 1c multiplies $E B_{x}$ values from Figure 1a bounded at zero by 2020 population estimates from the United Nations World Population Prospects 2019 to find the total number of surviving individuals bereaved by COVID-19 excess mortality as of July 2021. Note the different scales across panels. Results are shown where at least 1 percent of the group had a living relative of this type in February 2020. 
Our study underlines recent calls for more support to be given to individuals bereaved from COVID19 [3, 5, whether from their communities or governments. This is particularly urgent if, as recent evidence suggests, grief from COVID-19-associated bereavement is especially detrimental for survivors [10]. Moreover, these losses will reverberate in kin networks for generations to come. More research is needed on the impacts of excess bereavement on survivors, not just in the context of COVID-19, but for mortality crises past and present around the world.

\section{Materials and Methods}

Our analysis uses SOCSIM, a stochastic microsimulation platform that generates population-level genealogies using vital demographic rates as input [11, 12, 3. Our simulations use as input vital rates for the 1950-2035 period from the 2019 Revision of the United Nations World Population Prospects (medium scenario). To identify the effects of COVID-19 mortality on kinship structures, we run 200 microsimulations for each country, half representing a COVID-19 mortality case, and the other half representing a counterfactual scenario without excess mortality, with adjustments made using the Human Mortality Database's Short-term Mortality Fluctuations dataset (STMF) 22.

Some caveats should be noted. We assume zero international migration, and do not include marriage rates. Second, we do not consider within-country heterogeneity or clustering of excess mortality within groups or families, which means that our estimates are likely a lower bound for excess bereavement [13. Third, we assume demographic stability before 1950 in our simulations: this is a necessary assumption since reliable historical demographic data is not available for all countries studied. In the SI appendix, we apply formal demographic methods to historical Swedish data and show that the pre-1950 demographic stability assumption is unlikely to significantly affect the number of expected kin after 2015 for the types of kin considered in this paper.

As an additional robustness check, we compared our estimates of COVID-19-associated parental bereavement experienced by children to those obtained by Hillis et al. (2021), and found similarities in magnitude, despite differences in the age groups considered and methods employed.

\section{Acknowledgements and Funding Sources}

We thank Carl Mason, Kenneth Wachter, Tim Riffe, and Ugofilippo Basellini, as well as participants in the CenSoc Working Group, the Wittgenstein Centre Conference 2020, the Population Association of America Annual Meeting 2021, and the International Population Conference 2021.

We gratefully acknowledge support from the National Institutes of Health (2T32HD007275). The content is solely the responsibility of the authors and does not necessarily represent the official views of the National Institutes of Health.

\section{References}

[1] Goldstein JR, Lee RD (2020) Demographic perspectives on the mortality of COVID-19 and other epidemics. Proceedings of the National Academy of Sciences 117(36):22035-22041.

[2] Németh L, Jdanov DA, Shkolnikov VM (2021) An open-sourced, web-based application to analyze weekly excess mortality based on the Short-term Mortality Fluctuations data series. PLOS ONE 16(2):e0246663.

[3] Verdery AM, Smith-Greenaway E, Margolis R, Daw J (2020) Tracking the reach of COVID-19 kin loss with a bereavement multiplier applied to the United States. Proceedings of the National Academy of Sciences 117(30):17695-17701.

[4] Kidman R, Margolis R, Smith-Greenaway E, Verdery AM (2021) Estimates and Projections of COVID-19 and Parental Death in the US. JAMA Pediatrics 175(7):745-746.

[5] Hillis SD, et al. (2021) Global minimum estimates of children affected by COVID-19-associated orphanhood and deaths of caregivers: a modelling study. The Lancet 398(10298):391-402.

[6] Raker EJ, Zacher M, Lowe SR (2020) Lessons from Hurricane Katrina for predicting the indirect health consequences of the COVID-19 pandemic. Proceedings of the National Academy of Sciences 117(23):12595-12597. 
[7] Patterson SE, Verdery AM, Daw J (2020) Linked Lives and Childhood Experience of Family Death on Educational Attainment. Socius: Sociological Research for a Dynamic World 6:237802312097559.

[8] Wang H, Verdery AM, Margolis R, Smith-Greenaway E (2021) Bereavement from COVID-19, Gender, and Reports of Depression among Older Adults in Europe. The Journals of Gerontology: Series B. gbab132.

[9] Alburez-Gutierrez D, Mason C, Zagheni E (2021) The "Sandwich Generation" Revisited: Global Demographic Drivers of Care Time Demands. Population and Development Review 47(4):997-1023.

[10] Eisma MC, Boelen PA, Lenferink LIM (2020) Prolonged grief disorder following the Coronavirus (COVID-19) pandemic. Psychiatry Research 288:113031-113031.

[11] Hammel E, Hutchinson D, Wachter K, Lundy R, Deuel R (1976) The SOCSIM DemographicSociological Microsimulation Program: Operating Manual, Institute of International Studies. (University of California Berkeley).

[12] Wachter KW (1997) Kinship resources for the elderly. Philosophical Transactions of the Royal Society of London. Series B: Biological Sciences 352(1363):1811-1817.

[13] Vahabi N, Salehi M, Duarte JD, Mollalo A, Michailidis G (2021) County-level longitudinal clustering of COVID-19 mortality to incidence ratio in the United States. Scientific Reports 11(1):3088. 


\title{
SI Appendix: Extended Methods
}

\author{
Mallika Snyder, Diego Alburez-Gutierrez, Iván Williams, and Emilio Zagheni
}

February 16, 2022

\section{Adjusting Simulation Inputs for Excess Mortality}

In order to obtain simulation inputs, we first convert UNWPP 5-year mortality rates into monthly values assuming uniform mortality across the interval, except for the period from March 2020-June 2021. For this period, we employ weekly age and sex-disaggregated mortality data from the Human Mortality Database's Short-term Mortality Fluctuations dataset (STMF), to adjust the monthly rates in line with two possible mortality scenarios: a COVID-19 case using rates from the 2020-2021 period, and a counterfactual case using an average of the 2016-2019 monthly rates.

Since SOCSIM employs monthly vital rates, we first convert HMD STMF weekly data to monthly data by dividing each week's total by 7 and assigning each day thus generated to a month according to the ISO8601 standard, which is used by almost all of the countries in the STMF. We use this method to generate monthly exposures (from the STMF data) and death totals for 2016-2021. An average of 2016-2019 values forms our baseline of "expected" 2020-2021 mortality, and we use this to also calculate an annual average expected mortality rate by averaging across all monthly mortality rates. We then use this as the denominator of the ratio used in calculating the adjustment factors used in the two scenarios: in the COVID-19 case, the numerator is 2020-2021 monthly mortality rates, and in the counterfactual case, the numerator is the baseline 2016-2019 rate. Since the original rates to be adjusted are annual averages which do not account for seasonality, this approach allows for us to consider by how much mortality would have been expected to change in the months from March 2020-June 2021 in the absence of COVID-19 excess mortality, and by how much it increased above annual expected values during the period as a result of COVID-19 excess mortality.

\section{Sample Selection Criteria}

After running the simulations, as a robustness check we use simulation outputs to calculate excess mortality for the period by age group and compare this to equivalent estimates based on the STMF. In line with our input mortality adjustment factors, we calculate excess mortality as the average ratio across simulations of COVID-19 to counterfactual 65+ age-specific mortality rates for the March 2020June 2021 period; we then select only countries where the comparable value from the STMF data is within the normal-based $95 \%$ confidence interval derived from these simulations. In general, there is a high correlation between input and output (simulated) mortality rates; within the 11-country sample, the correlation for the $65+$ age group is .99, and for all 31 countries with data up till June 2021 included in the STMF dataset, the correlation is .98. This relatively conservative selection criterion formed the basis for our 11-country sample out of the 31 included in the HMD STMF: Bulgaria, Chile, Estonia, Finland, France, Latvia, the Netherlands, Norway, Portugal, Sweden, and the United Kingdom (obtained by combining mortality data from England and Wales, Scotland, and Northern Ireland).

\section{Investigating the pre-1950 Demographic Stability Assump- tion}

In this section, we consider the implications of assuming demographic stability before 1950. In particular, we examine the degree to which extrapolating the particular demographic characteristics of the 1950-1954 period into the past (including the baby boom) can bias the initial kinship structures for the countries we study.

One way to consider the impact of assuming demographic stability prior to 1950 on living kin is to think in terms of a particular kin relation, especially in terms of ancestors who experienced vital rates 
prior to the ego's birth. In the case of mothers, the probability that an ego aged $a$ in year $y$, from cohort $c=y-a$, had a living mother $M_{1}^{y}(a)$, can be approximated based on [1] with:

$$
M_{1}^{y}(a) \approx p^{\left[c-k_{c}\right]}\left(k_{c}, a\right),
$$

where $p^{\left[c-k_{c}\right]}\left(k_{c}, a\right)$ is the mother's probability of surviving $a$ years from age $k_{y-a}$ (the mean age of childbearing when ego was born), given cohort mortality risk for a person born in $\left[c-k_{c}\right]$.

Let us consider an ego born before 1950 who survives to subsequent years. This ego could be aged $a=30$ in $y=1960, c=1930$, living 20 years prior to 1950 and 10 after. Formula 1 can be factorized with the year 1950 as pivot:

$$
M_{1}^{1960}(30) \approx p^{\left[1930-k_{1930]}\right.}\left(k_{1930}, 20\right) * p^{\left[1930-k_{1930]}\right.}\left(k_{1930}+20,10\right)
$$

If rates (mortality and fertility) are assumed constant before 1950 (and female population structure by age too, which is relevant to the mean $k$ ), we should have a slightly different probability $M_{1}^{1960 *}(30)$, given that all the rates before 1950 now are related to that last observed year:

$$
M_{1}^{1960 *}(30) \approx p^{1950}\left(k_{1950}, 20\right) * p^{\left[1930-k_{1950]}\right.}\left(k_{1950}+20,10\right)
$$

We can identify two main effects that drive the difference between $M_{1}^{1960 *}(30)$ and $M_{1}^{1960}(30)$, given a direction of change for each one. Let us assume constant aging and a mortality decline. Before 1950 mothers would experience 1950 period rates instead of cohort ones, increasing mother's survival during ego's life. The second main effect goes in the opposite direction, given that the relation of maternal ages is $k_{1930}<k_{1950}$, survival chances decrease because of age (risk period starting in age $k_{1950}$ instead of $k_{1930}$ ) and a cohort effect (mortality schedule from [1930 $\left.-k_{1950}\right]$ cohort instead [1930 $\left.-k_{1930}\right]$ ). The net result will depend on the curvature of $l_{x}$, its change with time, and the slope of $k$ with time, not directly estimable without observed data or a parametric assumption on components. A similar approach can be made for older ancestors. For direct descendants both factors would also include cohort fertility rates.

This last case applies when ego is born before 1950 and is alive after. If she is born in 1950 or later, $y_{1} \geq 1950$, then this effect on living mothers would disappear. It also disappears for grandmothers if ego is born on $y_{2} \geq 1950+k_{1950}$, and for great-grandmothers if ego is born in $y_{3} \geq 1950+k_{1950}+k_{1950+k_{1950}}$, and so on. Of course, if ego is born after 1950, all living descendants would not be affected by the change. In general terms, the bias in constant rates before 1950 in living ancestors older than mothers (when $i>1$ ), will disappear for $M_{i}$ if ego is born at $y_{i+1} \geq y_{i}-k_{y_{i}-k_{1950}}{ }^{1}$. In a stable population context, when there is a sudden change in rates (here in 1950), changing the regime itself, this series could be useful to ensure that ancestors until $i$ degree belong to the new and latest regime.

We carried out separate analyses to understand the effect of assuming demographic stability before 1950 for a specific population. For a numeric example we applied kin estimation for an ego aged 30 (an age where it is not unlikely to have a surviving grandmother in low mortality countries, and also a living child [2]) using vital rates from Sweden and locating ego in successive years from 1950 to 2015.

We ran two sets of analyses: 'historical' analyses used the estimated historical rates for the 17511950 period and 'stable' analyses assumed that the 1950 demographic rates applied throughout the 1751-1950 period. Age-specific mortality rates (starting from 1751) come from the Human Mortality Database and fertility rates (starting from 1891) come from the Human Fertility Database. In all cases, we assumed demographic stability for fertility in the 1751-1891 period. We combined these data with official demographic projections of fertility and mortality provided by Statistics Sweden. Sweden is the ideal case for this analysis given its long time series of high quality demographic rates. Crucially, Sweden also experienced a moderate increase in fertility after WWII [3]. As such, the Swedish case would allow us to explore whether our stability assumption extrapolates the conditions of the post-WWII baby-boom into the past.

The results suggest that there is a general convergence in all the relatives types included in the study. The fact that older ancestors had later converged is consistent with the mentioned series $y_{i}$. In general terms the differences are null for all type of kin in year 2015 (last year of evaluation), when in 1950 we could see bigger differences, like $50 \%$ in cousins $9 \%$ in older sisters, $-50 \%$ in grand-mothers and $-80 \%$ in grand-daughters $\left(\left[\frac{\text { historical }}{\text { stable }}-1\right] * 100\right)$. In conclusion, at least in the Swedish case, no significant bias is expected on kin counts after 2015 because of assuming fixed rates before 1950 .

\footnotetext{
${ }^{1}$ The assumption of a concentrated fertility schedule around $k$ is necessary.
} 


\section{Validating our Simulations with Formal Methods}

To ensure that the simulated populations capture the kinship structure implied by the input demographic rates, we estimate a set of independent matrix kinship models with the same input data. We use an implementation of matrix kinship models [4] extended for populations with time-variant demographic rates, as implemented in the DemoKin $\mathrm{R}$ package (https://github.com/IvanWilli/DemoKin), to show that the estimates are consistent with the simulated kinship structures.

We report a comparison of estimates for living kin for a women in Sweden in July 2020 (the first wave of excess mortality), based on both microsimulation and formal methods. While the kin relations presented in the main text are for male and female kin, here we present only female kin in a singlesex population model so as to achieve comparability with the formal approach we present. The results suggest that there is considerable similarity between methods in terms of the results obtained, providing an important robustness check for our microsimulation-based results.

Kin counts are generally similar across the two methods. There are no significant differences for mothers, except for individuals aged $65+$, for whom SOCSIM estimates .07 living mothers and the GKP approach estimates .02 mothers in both the COVID-19 and counterfactual scenarios. In general, for a given method, population-level kin counts are fairly similar across the two scenarios, reflecting the relative brevity of the mortality shock considered as well as the large number of individuals included in the simulations. Differences in the number of living grandmothers are all under 0.15, with the largest discrepancies in the 30-44 age interval, followed by the 15-29 age interval. This is likely associated with the increased rarity of having a living grandmother at older ages, leading to more statistical variability in the SOCSIM estimates (although even older individuals might be so unlikely to have a living grandmother as to make estimates of kin availability and associated differences relatively small). The expected number of daughters is higher in GKP-based estimates for most age groups by about 0.1 to 0.18. Consistent with our expectations, we tend to find the largest discrepancies for the relative types that are less common overall. Our simulations have a limited size (for example, across simulation runs, there were around 6,500 women aged 30-44 in Sweden who survived the COVID-19 mortality period from March 2020 to June 2021) and, as a result, there is bound to be more uncertainty around the prevalence of relatively rare kin ties.

\section{Comparison to Other Published Estimates}

As an additional robustness check, we compare results from Hillis et al. (2021)'s modeled estimates of parental bereavement for children in countries from our sample. In general, our estimates are slightly lower, reflecting the lower age group endpoint (14 versus 17); most parentally bereaved children would likely be in the older portion of the age group. Hillis et al.'s estimate of 8,495 parentally bereaved children aged 0-17 in England and Wales alone by the end of April 2021 resembles our estimate of 6,989 children aged 0-14 for the United Kingdom as a whole for the same period, despite differences in the groups considered [5]. Similarly, we estimate 2,755 orphans aged 0-14 in France, compared to the Hillis et al. (2021) estimate of 4,064 orphans aged 0-17.

\section{References}

[1] Keyfitz N, Caswell H (2005) Applied Mathematical Demography. (Springer, New York).

[2] Alburez-Gutierrez D, Mason C, Zagheni E (2021) The "Sandwich Generation" Revisited: Global Demographic Drivers of Care Time Demands. Population and Development Review Advanced publication.

[3] Van Bavel J, Reher DS (2013) The Baby Boom and Its Causes: What We Know and What We Need to Know. Population and Development Review 39(2):257-288.

[4] Caswell H (2019) The formal demography of kinship: A matrix formulation. Demographic Research 41:679-712.

[5] Hillis SD, et al. (2021) Global minimum estimates of children affected by COVID-19-associated orphanhood and deaths of caregivers: a modelling study. The Lancet 398(10298):391-402. 